\title{
De la vanité des usages constants dans le monde du vin
}

Jean-Robert Pitte

\section{CpenEdition}

\section{Journals}

Édition électronique

URL : https://journals.openedition.org/geohist/2857

DOI : 10.4000/geohist.2857

ISSN : 2264-2617

Éditeur

Association française de la Revue de géographie historique

Référence électronique

Jean-Robert Pitte, " De la vanité des usages constants dans le monde du vin », Revue de géographie historique [En ligne], 19-20 | 2021, mis en ligne le 20 novembre 2021, consulté le 24 novembre 2022 URL : http://journals.openedition.org/geohist/2857 ; DOI : https://doi.org/10.4000/geohist.2857

Ce document a été généré automatiquement le 24 novembre 2022.

\section{c) $(9)$}

Creative Commons - Attribution - Pas d'Utilisation Commerciale - Pas de Modification 4.0 International - CC BY-NC-ND 4.0

https://creativecommons.org/licenses/by-nc-nd/4.0/ 


\title{
De la vanité des usages constants dans le monde du vin
}

\author{
Jean-Robert Pitte
}

\section{Introduction}

1 Les toutes premières politiques de délimitation des vignobles de qualité et d'établissement de méthodes exigeantes remontent au Moyen Âge et concernent les clos des abbayes, évêchés, chapitres et ducs ${ }^{1}$, puis rois en Bourgogne. Au XVIII ${ }^{e}$ siècle, trois princes européens établissent des aires de production et réglementent certaines pratiques viti-vinicoles, en particulier dans le domaine de l'encépagement. Ce sont les politiques établies par le Prince François II Rácóczi dans les premières années du XVIII ${ }^{e}$ siècle à Tokaj, par le grand-duc de Toscane Cosme III de Médicis en 1716 dans le Chianti et par le marquis de Pombal en 1756 dans la vallée du Douro. En France, à l'exception de la recherche d'excellence des châteaux bordelais, la situation est longtemps demeurée plus floue. Jusque dans les premières décennies $\mathrm{du} \mathrm{XX}^{\mathrm{e}}$ siècle, on produisait des vins mousseux appelés champagne en Val de Loire et, jusqu'à une date récente, nombreuses étaient les usurpations étrangères de ce vin de fête. Elles existent encore en Russie. On ne se privait pas dans les chais des négociants de Bourgogne et de Bordeaux de couper les vins locaux, parfois pâles et maigrichons, avec des vins dits «médecins ", venus de l'Hermitage et de toute la vallée du Rhône, d'Espagne, d'Italie ou d'Algérie. Au début du $\mathrm{XX}^{\mathrm{e}}$ siècle, un négociant bourguignon rendant visite à Madame Le Saint, belle-mère du baron Le Roy et propriétaire du Château Fortia à Châteauneuf-du-Pape, crut lui faire plaisir en lui déclarant ${ }^{2}$ : "Vous êtes devenus, à Châteauneuf, notre succursale », ce à quoi elle lui répliqua : «Vous vous trompez, Monsieur, nous sommes la maison-mère ! » 


\section{I-Grandeur passée et inadaptation actuelle des lois françaises de protection de l'origine}

2 La loi du 5 août 1908 relative à la délimitation des régions viticoles revendiquant une appellation d'origine est le premier texte juridique qui évoque les « usages locaux constants ». La loi du 6 mai 1919 précise que les vins d'origine doivent respecter « les usages locaux, loyaux et constants", ce qui exclut les coupages, qui sont encore monnaie courante à cette époque et ne cesseront pas aussitôt. La loi du 22 juillet 1927 grave ces règles dans le marbre en établissant un lien entre un vin et un territoire ou un terroir donné dont la taille peut aller de la parcelle, à la commune ou à la région. Le décret-loi du 30 juillet 1935 le confirme et donne tout pouvoir à un Comité national des appellations d'origine des vins et des eaux-de-vie (CNAO) qui se prononce sur les propositions des syndicats locaux et dont le premier président est le sénateur Joseph Capus, ancien ministre de l'agriculture, qui a travaillé en étroite complicité avec le baron Le Roy de Boiseaumarié, créateur du syndicat des vignerons de Châteauneuf-duPape et de celui des Côtes-du-Rhône. En 1947, le CNAO devient Institut national des appellations d'origine des vins et des eaux-de-vie, (INAO), présidé par le baron Le Roy ${ }^{3}$, puis en 2007, Institut national de l'origine et de la qualité.

3 D'évidence, ces réglementations de plus en plus précises et encadrées, parfois un brin pointilleuses, comme il se doit en France, ont permis depuis un siècle des progrès immenses dans la recherche de la qualité des vins. Jamais on n'a élaboré d'aussi bons vins qu'aujourd'hui. Ils sont le plus souvent précis, authentiques ou sincères, c'est-àdire typiques de leur terroir d'origine, digestes et ce n'est qu'un début, car la viticulture et les vinifications progressent constamment. Cette politique a d'ailleurs été imitée dans toute l'Europe - qui gère depuis 1992 la politique des Appellations d'origine protégée (AOP) - et toujours davantage dans la plupart des grands vignobles du Monde, à l'exception notable de la Chine où les vins garantis d'origine produits dans le pays sont encore rares. Rappelons que le vote et l'application de ces lois ne coula pas de source. Des oppositions farouches se manifestèrent. En premier lieu, celles du négoce de tous les vignobles de France (Bordeaux, Bourgogne, en particulier) et celui des villes de Sète et de Paris (Bercy) qui, du fait de l'interdiction des coupages, perdaient une grande partie de leur raison d'être et durent adapter leur activité. Les pratiques douteuses perdurèrent fort tard et de sévères condamnations touchèrent encore de célèbres maisons, comme Cruse à Bordeaux en 1973 ou Labouré-Roi à Nuits-SaintGeorges en 2012. Par ailleurs, s'opposèrent à cette évolution certains grands propriétaires de châteaux bordelais attachés à leur marque et à la liberté de vendre ce qu'ils voulaient sous leur propre étiquette. Ce fut, par exemple, le cas du duc de La Trémoille, député-maire de Margaux - républicain de gauche - et alors propriétaire du célèbre Château Margaux qui déclara à l'Assemblée le 13 novembre 1913 :

«Peut-on contester au détenteur d'un vignoble le droit de faire du nom de ce vignoble l'usage qu'il lui plaît? Si l'attribution du nom d'origine d'un produit est subordonnée à certaines qualités, il est porté atteinte au droit de propriété. »

C'est ce qui explique la volonté des propriétaires concernés de voir le classement de 1855 inscrit par l'UNESCO sur la liste du patrimoine immatériel mondial. Établi par la Chambre de Commerce, sur des critères purement commerciaux et sans référence à des obligations liées à la viticulture ou à la vinification, il ne reflète que très partiellement la géographie des terroirs bordelais, ne serait-ce que parce qu'il exclut les Graves, à 
l'exception de Haut-Brion, toute la rive droite (Saint-Émilion, Pomerol) et que SaintEstèphe, alors plus difficilement accessible au négoce bordelais, ne comporte que cinq châteaux sur les 56 de la liste du Médoc. Par ailleurs, tous les critiques et les amateurs savent que la hiérarchie en cinq crus est loin de correspondre tous les ans au verdict de la dégustation.

5 Ne voulant pas être en reste, les vignerons de Saint-Émilion qui, comme ceux de Pomerol, s'étaient depuis toujours passés de classement, voulurent un siècle plus tard imiter la rive gauche et hiérarchiser les crus, ce que le décret de 1936 n'avait pas envisagé. En 1955, leur syndicat établit un classement révisable distinguant des simples saint-émilion, trois catégories supérieures : les saint-émilion grands crus, les saintémilion premiers grands crus classés B et les saint-émilion premiers grands crus classés A, la strate supérieure ne comprenant que deux châteaux : Ausone et Cheval Blanc. Le classement fut révisé six fois et, en 2006, donna lieu à d'intenses polémiques et à des procès intentés par des propriétaires dont le cru avait été déclassé, par exemple le Château Corbin-Michotte de Jean-Noël Boidron. Pour éviter que cette situation se reproduise, en 2012, l'INAO se chargea du classement et furent promus deux nouveaux premiers grands crus classés $\mathrm{A}$ : le Château Angélus et le Château Pavie appartenant à deux influents propriétaires, très actifs dans le monde du vin et dans les médias: Hubert de Boüard et Gérard Perse. Les critiques furent aussi nombreuses qu'en 2006 et, coup de tonnerre, à l'occasion de la révision de 2021 pour laquelle les épais dossiers de candidatures devaient être déposés le 30 juin, Ausone et Cheval Blanc décidèrent de ne pas se soumettre au verdict et de sortir du classement. D'aucuns affirment pour l'expliquer que ${ }^{5}$ «la marque est plus forte que le classement. Cheval Blanc et Ausone peuvent s'en passer. » C'est sans doute vrai, mais pour les premiers grands crus classés $B$, gravir une marche est une opportunité d'augmenter la notoriété, la demande et donc les prix. En 2021, de nouveaux premiers grands crus A risquaient de rejoindre le petit peloton de tête et les vins des deux châteaux historiques risquaient de perdre de leur valeur, du moins leurs propriétaires l'ont-ils craint. On comprend les efforts de communication réalisés par certains châteaux. Force est de constater qu'ils paient davantage que les efforts qualitatifs, tels ceux que réalisa en vain pendant des décennies feu Thierry Manoncourt, propriétaire du Château Figeac, toujours classé premier grand cru B, et que poursuivent aujourd'hui Marie-France Manoncourt et sa famille. Il était reproché à son vin de comporter une proportion de cabernet-sauvignon supérieure à la moyenne de Saint-Émilion et donc de trahir la typicité de l'appellation dans laquelle domine le merlot. Rien de comparable en Médoc où l'encépagement original du Château Pichon-Longueville Comtesse de Lalande (60\% seulement de cabernet-sauvignon, contre $78 \%$ à Mouton-Rothschild, 30\% de merlot, $5 \%$ de cabernetfranc et $5 \%$ de petit verdot) n'a jamais posé le moindre problème quant à sa place de second dans le classement de 1855 que personne ne se risquerait à remettre en cause car il y aurait trop d'inconvénients pour les vénérables crus distingués à cette époque. Seule entorse à celui-ci : l'accession de Mouton-Rothschild au rang de premier en 1973, sous la discrète pression de Georges Pompidou qui avait travaillé naguère pour la banque.

6 Le résultat du retrait des deux ténors de Saint-Émilion est que le principe du classement est suffisamment discrédité pour qu'il soit sans doute abandonné et que le fonctionnement de l'appellation revienne au statu quo ante. À Pomerol dont le prix des bouteilles varie en primeur d'environ $20 €$ (Château de Sales) à $1000 €$ (Château Pétrus), 
personne ne se plaint du flou artistique qui laisse libre cours aux lois du marché, c'est à dire aux talents conjugués des domaines et des négociants confrontés au goût, au désir et au pouvoir d'achat des consommateurs. Éric de Rothschild et sa famille espèrent bien un jour améliorer la qualité et la notoriété du Château L'Évangile afin que les prix de ses vins atteignent ceux de Pétrus ${ }^{6}$. Cela semble, au fond, une situation bien plus saine et paisible et nul ne souhaite entrer dans l'engrenage d'un classement, source de conflits, de jalousies et d'incessants coups bas.

7 Décidément, la culture viticole bordelaise est en délicatesse avec le terroir, même si feux les géographes Henri Enjalbert et René Pijassou, dans tous leurs travaux, tentèrent de hiérarchiser les crus - et de justifier le classement de 1855 - en fonction des sols et de l'épaisseur des graves, position qui ne résiste que partiellement à l'analyse. N'oublions pas que Mouton Cadet désigna au début des années 1930 le second vin du château Mouton-Rothschild et qu'il s'agit aujourd'hui d'une simple marque sans lien avec le terroir prestigieux de Pauillac, assemblage de bordeaux génériques achetés dans les coopératives de la région et dont environ 12 millions de bouteilles sont chaque année mises en marché. Elles sont vendues entre 10 et $13 €$ la bouteille en France, alors que l'on trouve dans la grande distribution d'honorables bordeaux à moitié prix, mais sans la prestigieuse référence aux Rothschild qui, il est vrai, ont déployé beaucoup d'énergie depuis un siècle et demi pour produire quelques-uns des meilleurs vins du monde. Sur les étiquettes des crus classés de la rive gauche du vignoble du Bordelais, le nom de l'appellation communale n'apparait qu'en toutes petites lettres, voire pas du tout, comme c'est le cas pour Yquem. Après le rachat de la majorité des actions de ce château par le groupe LVMH de Bernard Arnault, la mention « Sauternes » réapparut entre 2001 et 2011, car il fallait meubler la plus sobre étiquette du Bordelais et remplacer la mention Lur-Saluces, nom de l'ancienne famille propriétaire. Depuis, elle a été supprimée et l'étiquette est ne porte plus que la mention Château d'Yquem et la couronne historique du blason des Lur-Saluces redessinée ${ }^{7}$. La philosophie des châteaux bordelais est si éloignée du primat du terroir qu'il est fréquent que certains propriétaires acquièrent des vignes en périphérie de leur domaine et augmentent ainsi, sans qu'aucune autorité n'y trouve à redire, leur production de premier et de second vin. En effet, les $\mathrm{AOC}$ ne se réfèrent en Bordelais qu'à des provenances communales ou régionales. Il existe même des "châteaux" dont l'étiquette porte le nom d'une demeure prestigieuse bien réelle, mais qui assemblent les vins de domaines éloignés de plusieurs kilomètres les uns des autres. C'est le cas du château Fombrauge, grand cru de Saint-Émilion, propriété de Bernard Magrez, dont les 58 hectares de vignes sont répartis sur les communes de Saint-Christophe-des-Bardes, Saint-Etienne-de-Lisse et Saint-Hippolyte, dans le prolongement du plateau et des coteaux de Saint-Émilion. Les sous-sols, sols et expositions y sont extrêmement divers.

8 La mise au point des appellations d'origine contrôlée dans la première moitié du $\mathrm{XX}^{\mathrm{e}}$ siècle fût une affaire de bons vignerons qui ne tombaient pas dans la pratique des coupages se déroulant en aval de leur travail, dans le secret des chais du négoce. C'est la raison pour laquelle, la hiérarchie bourguignonne en appellations régionales, villageoises, premiers crus et grands crus fut, comme on l'a dit, combattue par les négociants. De ce fait, les communes où ils étaient les mieux implantés, Nuits-SaintGeorges, Beaune, Meursault, ne comptent pas de grands crus, bien que certains climats de ces terroirs l'auraient largement mieux mérité que certaines appellations tenues par les vignerons et qui furent délimitées avec un certain laxisme (Corton, par exemple). Volnay est une exception: le marquis Sem d'Angerville, président du syndicat des 
vignerons et défenseur du décret-loi Capus, aux côtés du baron Le Roy, s'opposa à ce que des grands crus soient délimités dans sa commune, non pas en raison d'une qualité inférieure des vins, mais par scrupule: il était en effet propriétaire des meilleurs climats - dont le clos-des-ducs - et lui seul aurait bénéficié des appellations les plus prestigieuses dans lesquelles serait entré tout son domaine et quasiment lui seul ${ }^{8}$. C'est ce souci d'authenticité et ce perfectionnisme, sur lequel se greffe un discours élaboré d'effacement du travail des vignerons face aux données prétendument intangibles du terroir, qui se poursuit depuis lors en Bourgogne et qui a abouti en 2015 à l'inscription par l'UNESCO des 1247 climats de Bourgogne (en réalité seulement une partie de la Côte d'Or et quelques terroirs de Saône-et-Loire) sur la liste du patrimoine mondial. Aubert de Villaine, co-gérant du plus célèbre domaine de Côte d'Or, celui de la Romanée-Conti, en a été l'infatigable artisan, avec l'aide de Guillaume d'Angerville, petit-fils du marquis Sem. Cette décision aurait aussi bien pu être prise dans le cadre de la liste du patrimoine immatériel, car c'est d'abord une philosophie du vin de terroir qui a été considérée comme possédant une valeur universelle et qui, de fait, est de plus en plus imitée hors de la Bourgogne, en France (les 51 grands crus d'Alsace, les sélections parcellaires de Michel Chapoutier à l'Hermitage) ou à l'étranger (certains vignobles californiens ou sud-africains, par exemple).

Notons enfin à propos des AOC accordées dans le prolongement de la loi de 1936 le rôle de quelques propriétaires éclairés qui avaient su faire de leurs vins des crus si remarquables qu'ils bénéficièrent d'appellations contrôlées pour eux seuls : Château Grillet, sur le coteau de Condrieu, La Coulée de Serrant, au cœur des savenières, La Romanée-Conti, La Tâche, La Romanée et la Grande Rue à Vosne-Romanée, le Clos de Tart et, à une parcelle près, le Clos de Lambrays à Morey-Saint-Denis. Pour surprenants qu'ils soient, ces monopoles n'ont pas été accordés par complaisance et sont parfaitement conformes à l'esprit de la loi. Cela dit, certains propriétaires possédant des vignes dans des appellations voisines moins prestigieuses ont énormément progressé et réussissent certaines années ou, parfois, sur des périodes plus longues, des vins d'une qualité supérieure à celle des vins issus de ces niches. Jusqu'à ces dernières années, le fait était patent à Condrieu ; le rachat du Château Grillet par François Pinault en 2011 justifiera peut-être le choix de l'INAO en 1936. C'est un sujet ô combien délicat, en particulier pour les critiques. Cela fait rêver certains bons vignerons réputés qui aspirent à voir reconnu leur talent par une AOC spécifique. C'est le cas de Jean-Pierre Amoreau au Château Le Puy dans l'appellation peu connue Francs-côtes-de-bordeaux. Grâce à son talent viti-vinicole et médiatique, il est parvenu à vendre ses vins à un prix plus qu'honorable dans le monde entier. Il a même bénéficié du succès du manga japonais Gouttes de Dieu, dans lequel son vin est considéré comme le meilleur d'une impressionnante cohorte de bouteilles prestigieuses. Aujourd'hui, il bataille pour que le cœur de son terroir bénéficie d'une appellation spécifique, sans succès jusqu'à présent ${ }^{9}$.

Malgré ses ambiguïtés et son bureaucratisme, la politique de contrôle de l'origine menée depuis un siècle a sans nul doute entrainé des effets globalement très positifs, mais il est aujourd'hui permis de poser la question de l'intérêt de maintenir l'un des trois volets des textes qui l'encadre : celui des usages constants. Un certain nombre de vignerons - et non des moindres - ne les comprennent plus et préfèrent quitter le navire pour produire les vins qu'ils souhaitent et qu'apprécient leurs clients. 


\section{II-Repenser la réglementation sur les appellations d'origine}

11 Depuis plusieurs décennies, la planète des vins connaît de grands bouleversements. Il y a d'abord de plus en plus d'amateurs sur tous les continents. La consommation augmente partout, sauf dans les vieux pays viticoles d'Europe où, de préférence à l'eau qui était douteuse, on ne buvait naguère que du vin à table, la qualité de celui-ci variant en fonction des régions, mais surtout des revenus et de l'éducation du palais des consommateurs. Il s'agissait essentiellement de pays catholiques ou orthodoxes où le vin revêt un immense prestige, car il est, avec le pain, l'un des éléments de base de la transsubstantiation eucharistique. Aujourd'hui, dans le monde entier, à l'exception des pays musulmans, le vin est symbole de culture, de convivialité, de prospérité économique, d'intégration réussie au sein de la mondialisation. La plupart des pays plantent des vignobles ou augmentent leur superficie, même là où les conditions environnementales ne s'y prêtent guère (Pologne, Danemark, Grande-Bretagne, Canada, Amazonie brésilienne, Gabon, Thaïlande, île de Hokkaido au Japon, Polynésie, etc. $)^{10}$, avec des résultats parfois fort honorables. L'expansion de la viticulture de qualité est en cours aux États-Unis, en Argentine, au Chili, en Afrique du Sud, en Nouvelle-Zélande, en Australie et ailleurs. Nul doute que la Chine qui possède d'ores et déjà le deuxième vignoble mondial (dont une partie importante est tournée vers la production de raisin de table) et qui est le $10^{\mathrm{e}}$ producteur de vin, quasiment à égalité avec l'Allemagne, le Chili et l'Afrique du Sud, abandonnera vite le productivisme pour se tourner vers la qualité, à mesure que s'affinera le goût de ses consommateurs. Pour un pays comme la France qui a longtemps raisonné et agi comme s'il possédait le monopole de la production des grands vins, cela oblige les viticulteurs et le négoce à des efforts, voire à une remise en cause des vieilles habitudes afin de maintenir à l'étranger le désir de boire des vins français, d'autant que leurs prix sont parmi les plus élevés au monde.

Face à une concurrence internationale accrue, la seule solution est de poursuivre l'amélioration de la qualité et de renforcer la typicité de toutes les catégories de vins tout en pratiquant des prix de vente correspondant aux possibilités et aux attentes du marché. Soulignons à ce sujet qu'il y aura toujours un marché pour des bouteilles très onéreuses, dotées d'une image prestigieuse susceptible de faire rêver. Il faut simplement que la perfection soit au rendez-vous... Il y a des amateurs qui acceptent de débourser $32480 €$ (prix constaté en 2019) à la boutique hors-taxe de l'aéroport Charlesde-Gaulle, pour une bouteille de romanée-conti 1997, soit 43306,67€ le litre ! Ce marché de niche existe aussi en joaillerie, en horlogerie ou, bien entendu, dans le monde de l'art. Mais, qu'on ne se méprenne pas, les prix de vente rémunérateurs, élevés, voire astronomiques, ne concernent qu'une infime partie des productions du vignoble français : les crus classés du Bordelais, la plupart des vins de Côte d'Or quelle que soit leur appellation, les champagnes de grandes maisons, particulièrement leurs cuvées millésimées ou spéciales, les vins de quelques appellations réputées (château-chalon, condrieu, hermitage, côte-rôtie, châteauneuf-du-pape, de rares cuvées provençales, par exemple). À l'exception de quelques domaines qui ont su se faire connaître à l'étranger, la plupart des vins d'Alsace, de Loire, du Mâconnais et du Beaujolais, des Côtes-durhône, du Languedoc, de la moyenne Garonne se vendent à des prix plus que 
raisonnables pour les consommateurs, parfois à peine rémunérateurs pour les vignerons dont un certain nombre jettent l'éponge, faute de trouver un successeur.

Il ne s'agit nullement de remettre en cause l'excellent principe des appellations d'origine contrôlée qui assure aux vins français une notoriété qu'ils ne pourraient obtenir avec le seul nom de «vin français » auquel on ajouterait celui du cépage ou des cépages utilisés. Comme le disait l'ancien président de l'INAO, René Renou ${ }^{11}$ :

«Je crois toujours aux appellations, au concept d'appellation. Je suis absolument convaincu que c'est la seule alternative qui nous reste aujourd'hui pour survivre dans le milieu du vin et dans le marché mondialisé du vin. Ou on va chercher à produire des types de vins normatifs, ou on va vers des vins à forte identité culturelle et à forte spécificité. C'est là le challenge des AOC. Car si nous devions aller vers du normatif, où seule la qualité objective sera un critère de sélection pour le consommateur, je ne suis pas sûr que nous soyons capables de nous battre contre les vins du Nouveau Monde. Car nous devrons aller vers une banalisation et une standardisation qui est un rapport de force purement économique où la structure du vignoble français ne pourra pas s'affirmer. Ce qui fait la force des appellations françaises, c'est l'expression d'un terroir, cause de l'appellation d'origine. »

Le concept de terroir est éminemment français et, par bonheur, est en train de devenir contagieux un peu partout dans le monde. Au terme d'innombrables réunions parfois tendues, la Commission culturelle de l'INAO $^{12}$ a adopté lors de sa réunion du 29 novembre 2005 la définition suivante :

«Un terroir est un espace géographique délimité, où une communauté humaine, consommateurs inclus, construit dans la durée un savoir collectif de production fondé sur un système d'interactions entre un milieu physique et biologique, et un ensemble de facteurs humains. Dans un terroir, les techniques mises en jeu révèlent une originalité, confèrent des caractéristiques particulières, et engendrent une réputation, pour un produit originaire de cet espace géographique.»

15 Face à des pays qui privilégient encore la marque, le cépage et la standardisation, la segmentation en quelque 363 AOP et 74 IGP de vins ${ }^{13}$ est une chance pour la France, mais cela risque de ne pas durer éternellement, car d'autres pays suivent une évolution comparable. Face à cette tendance, la gestion des appellations doit évoluer. Elle doit d'une part être beaucoup moins tatillonne et, d'autre part, de plus en plus rigoureuse sur la qualité et, surtout, la typicité. Le respect scrupuleux des traditions héritées de l'histoire ancienne ou récente ne doit plus être un critère primordial. Un patrimoine figé meurt (les monuments historiques et les paysages, les recettes de cuisine, les langues et les littératures, etc.). Le faire vivre, c'est accepter son évolution, bien entendu dans le souci d'un perfectionnement. D'ailleurs, en les analysant bien, les soidisant usages constants ne sont souvent que des choix récents, au mieux postphylloxériques.

16 Ainsi en est-il de l'encépagement. Il est aujourd'hui gravé dans le marbre des décrets d'appellations et se limite à une cinquantaine de variétés principales, alors qu'il s'en cultivait des centaines avant le phylloxera ${ }^{14}$. Au recensement viticole de 1958 , on en dénombrait encore 272 dans le seul département de l'Ain qui était, il est vrai, une sorte de vignoble fossile dont la notoriété des vins était très faible ${ }^{15}$. La pratique de la complantation de plusieurs cépages était naguère courante. Il existe à Segrois dans les Hautes-Côtes de Nuits une vieille vigne de passetoutgrain appartenant au domaine de Montmain encore complantée de pinot noir et de gamay. Le millésime 1976 qu'elle produisit était du niveau des premiers crus de la Côte d'Or. L'Alsace produit presqu'exclusivement aujourd'hui des vins monocépages (une douzaine de variétés), 
comme le prévoient les décrets d'appellations, y compris dans les 51 grands crus, à l'exception de l'edelzwicker, du gentil ou du crémant, pour lesquels l'assemblage est autorisé. Cette pratique restrictive n'est nullement ancienne comme aime à le démontrer, documents à l'appui, l'inventif vigneron de Bergheim, Jean-Michel Deiss ${ }^{16}$ qui a remis à l'honneur dans ses terroirs classés en grands crus, non pas l'assemblage de cuvées monocépages, à la manière bordelaise ou champenoise, mais le complantage dans la même parcelle de plusieurs cépages. Cela donne des vins d'une grande complexité qui, selon lui, expriment pleinement les spécificités pédologiques du cru, alors que le monoencépagement privilégie le goût des variétés dont il est vrai que la personnalité est forte et contrastée en Alsace. Leur caractéristique qui peut gêner certains consommateurs, c'est qu'ils possèdent aussi une dose de sucre résiduel plus élevée que la moyenne régionale, non pas d'abord en raison du réchauffement climatique, mais du fait que la vendange ne peut s'effectuer que lorsque les cépages tardifs sont mûrs et que, par conséquent, les précoces sont surmûris. Ébranlée par les arguments historiques présentés par Jean-Michel Deiss, l'INAO tolère qu'il utilise les appellations en usage en Alsace, en particulier les noms des grands crus qu'il exploite.

La question des cépages autorisés est un autre sujet de tension depuis des années entre l'INAO et un nombre croissant de vignerons. Pendant longtemps, l'administration s'est montrée intraitable à l'égard des innovateurs, même lorsqu'ils se référaient à des traditions antérieures au $\mathrm{XX}^{\mathrm{e}}$ siècle. L'un des exemples les plus célèbres est celui du domaine de Trévallon à Saint-Étienne-du-Grès, sur le flanc nord des Alpilles, dans l'aire AOC «Baux-de-Provence », une appellation bénéficiant depuis 1995 du nom prestigieux d'un haut-lieu de la Provence et qui regroupe aujourd'hui 15 domaines. Le néovigneron Éloi Dürrbach créa de toutes pièces le domaine de Trévallon à partir de 1973, mais, s'appuyant sur les écrits de Jules Guyot en 1868, il décida d'élaborer des vins rouges à partir d'un encépagement partagé à égalité entre la syrah et le cabernetsauvignon. Or, ce dernier avait depuis longtemps disparu de Provence et n'était plus alors autorisé. Pendant des années les vins ne purent être commercialisés que sous l'appellation ordinaire - presque infâmante - de "vin de pays des Bouches-du-Rhône ». Cependant, leur excellence permit à Éloi Dürrbach de les vendre, à des prix très supérieurs à la moyenne régionale, à des importateurs du monde entier, à des cavistes réputés et même aux restaurants triplement étoilés de la région comme l'Oustau de Baumanière aux Baux-de-Provence ou Le Petit Nice à Marseille. Aujourd'hui, Trévallon ne peut toujours pas vendre ses vins en AOP sous l'appellation "baux-de-Provence ", mais a été autorisé à utiliser l'«IGP Alpilles », petite concession qui fait évidemment sourire les nombreux inconditionnels de ses vins si réussis. Gageons que cela laisse de marbre Éloi Dürrbach qui n'en n'a cure et pourrait réussir, même s'il n'était autorisé qu'à l'appellation « vin de France ».

18 Cela avait naguère réussi à Aimé Guibert qui avait créé à Aniane en Languedoc le domaine Mas de Daumas-Gassac. En s'appuyant sur des études d'Émile Peynaud et René Pijassou, lesquels lui avaient recommandé de planter en partie son terroir de cabernetsauvignon, il avait tenté de persuader le marché qu'il produisait des vins du niveau des grands crus bordelais et vendait du «vin de table » à des prix stratosphériques. Ce faisant, il avait bataillé avec succès pour que l'américain Mondavi de vienne pas créer un domaine concurrent aux portes du sien. Hélas pour Aniane qui a perdu ainsi une cinquantaine d'emplois ${ }^{17}$ ! En attendant, nombre de vignerons talentueux et disposant souvent d'une notoriété mondiale sont en train de quitter le navire de l'AOC pour basculer de leur plein gré en IGP ou en « Vin de France », appellations qui leur donnent 
bien plus de latitude pour interpréter leurs terroirs de la manière la plus juste et la plus originale possible ${ }^{18}$. La goutte d'eau qui a fait déborder le vase et balayé leur docilité a souvent été un refus d'appellation par l'INAO pour l'une ou l'autre de leurs cuvées. Citons: Dominique Hauvette aux Baux-de-Provence qui rejoint son mentor Éloi Dürrbach, feu Laurent Vaillé au domaine de La Grange des Pères en Languedoc, Mark Angeli en Anjou, Louis-Benjamin Dagueneau à Pouilly-sur-Loire, François Chidaine à Vouvray (dont le chai est à Montlouis, ce qui chiffonne l'INAO !), Pascal Amoreau dans les Francs-Côtes-de-Bordeaux, Jean-Charles Abbatucci en Corse (pour usage de vieux cépages non prévus dans les décrets), Franck Pascal à Bergerac (exclu pour avoir utilisé une année par nécessité une bouteille non conforme !).

Ébranlée par des demandes d'extension du nombre des cépages autorisés, émanant de vignerons de plus en plus nombreux, dans toutes les régions de France, l'INAO est en train de revoir lentement ses positions. Le grand vigneron de Gaillac Robert Plageoles ${ }^{19}$ est parvenu à convaincre le syndicat local, puis l'INAO, de l'intérêt d'utiliser de vieux cépages oubliés depuis des lustres : la muscadelle, l'ondenc, le prunelart. Les délicieux vins issus de ce dernier furent d'abord vendus sous l'étiquette «vin de pays ». Depuis 2008, ils bénéficient de l'AOC. Robert Plageoles est aussi le découvreur et le protecteur d'un vignoble de vignes «sauvages» dans la forêt de la Grésigne. Ces variétés pourraient un jour venir enrichir le patrimoine génétique des vignes actuelles pour leur permettre de résister aux maladies et surtout donner des vins totalement nouveaux créant ainsi un lien avec le vignoble antique de cette région, l'un des plus anciens de Gaule. Enfin, il remet à l'honneur certaines pratiques viticoles tombées dans l'oubli et qui permettent d'obtenir des vins de grande originalité qui ont su conquérir de nombreux amateurs: les vendanges tardives (AOC depuis 2011), le mauzac mousseux, le vin de voile, le vent d'Autan à base d'ondenc. Ce dernier fut servi en 2005 par le président J. Chirac à un déjeuner de chefs de gouvernement se tenant à Toulouse. Signalons une expérience comparable, mais qui n'entre pour l'heure dans aucun projet commercial, celle de l'association qui gère le vignoble du château de Chareil-Cintrat (Allier), en étroite collaboration avec le Centre des Monuments nationaux, propriétaire du château et des terres qui l'entourent. Elle maintient les anciennes conduites de la vigne et, surtout, cultive plusieurs cépages de la région dont certains ont aujourd'hui disparu : saint pierre doré, meslier saint françois, romorantin, melon, aligoté, tressalier (cépage original de Saint-Pourçain), pinot chardonnay, sauvignon, pinot gris, pinot blanc. Tous les raisins sont vinifiés ensemble et donnent une belle cuvée appelée «Assemblage des anciens cépages du Pays Saint-Pourcinois». Elle ne porte que l'appellation "Vin de France » et présente davantage d'originalité que beaucoup de vins produits dans l'aire de l'AOC Saint-Pourçain. L'INAO devrait bien davantage s'intéresser à cette modeste et sympathique initiative.

Dans la famille des vignerons archéologues qui veulent faire revivre les cépages et les pratiques de jadis, certains jouent cavalier seul et ne souhaitent pas bénéficier de l'AOC qui ne leur apporte rien et, au contraire, dilue leur originalité. Ainsi, en est-il de Liber Pater, le vin «de garage " que produit Loïc Pasquet à Landiras, près de Bordeaux, actuellement le plus cher du monde, vendu en primeur $30000 €$ la bouteille, désormais sous l'appellation "Vin de France " alors qu'il bénéficiait de l'AOC Graves jusqu'en 2010. Son créateur s'explique ${ }^{20}:$ " Il y a seulement 250 bouteilles de cette cuvée à la vente, et c'est la première fois qu'on est $100 \%$ en franc-de-pied, donc sur un assemblage de cépages historiques de Bordeaux non-greffés, comme la petite-vidure, le petit verdot, le malbec principalement, pour l'assemblage de ce 2015. Cette cuvée, c'est un 
peu comme une œuvre d'art éphémère, les gens veulent goûter ce qui n'existe plus, vivre une expérience unique. Pour fixer ce tarif, j'ai vu les demandes sur ce millésime, j'ai vu ce que j'avais à offrir, et les gens ont acheté. C'est le principe de l'offre et de la demande, tout simplement. » Et Loïc Pasquet d'ajouter avec une feinte candeur qui a de quoi agacer les viticulteurs des Graves : « Le marketing, je n'y connais rien, ce n'est pas mon métier. Et puis je ne suis pas dans une compétition avec la Romanée. Je connais bien Aubert de Villaine, nos vins sont sur des positionnements différents. La Romanée c'est 4000 bouteilles, Liber Pater 250, la Romanée est un monocépage, Liber pater un multicépages... Je dirais que la Romanée c'est un peu du Chopin, et Liber Pater du Mozart. L'un est-il mieux que l'autre ? Je n'en sais rien, ce sont les goûts de chacun, moi j'aime les deux. J'aime bien me lever en écoutant Mozart et me coucher en écoutant Chopin. » Bien entendu, cet incroyable succès s'appuie sur une communication subtile, de nombreux entretiens accordés à la presse française et internationale et sur une $\mathrm{BD}^{21}$ dans laquelle Loïc Pasquet justifie l'adoption comme marque de son vin du nom du dieu romain assimilé à Bacchus (p. 106) : «Dans l'Antiquité, plusieurs dieux imposent aux hommes le respect de l'environnement. Le monothéisme, quant à lui, impose la soumission du monde à l'homme. "! La conclusion est que lorsqu'un vigneron, habile vendeur, produit des vins originaux, le fait que ceux-ci ne soient pas reconnus en AOC/ AOP n'est nullement un handicap, au contraire. Il y a toujours et partout des amateurs qui aiment servir à leur table des vins atypiques et rares, parfois dotés de vraies qualités intrinsèques, parfois seulement d'une étiquette atypique. L'absence de reconnaissance administrative intrigue et augmente leur attractivité.

21 La question des usages constants en matière d'encépagement est aussi en train de prendre un nouveau tournant en raison du réchauffement climatique. Certains vignerons des Hautes-Côtes de Beaune et de Nuits, voués naguère à l'aligoté et au gamay, élaborent aujourd'hui de superbes cuvées à partir du chardonnay et du pinot noir. Constatant que leurs collègues de la Côte de Beaune et de la Côte de Nuits font face à des problèmes de grillage de leurs raisins, de surmaturité et de déficit d'acidité qui leur demandent de constantes adaptations, ils affirment discrètement que ce sont eux qui, bientôt, élaboreront les grands crus de Bourgogne! Ce discours était le morceau de bravoure de feu Bernard Hudelot, vigneron à Villars-Fontaine. Les immenses progrès réalisés par les vignerons du Haut-Languedoc, ceux des Terrasses du Larzac, par exemple, par rapport à ceux des zones basses ne peuvent que les conforter dans leurs espérances. Dans le Sud-Ouest, malgré les réticences de l'INAO, certains cépages tardifs sont en cours de réintroduction; le bouysselet à Fronton, par exemple ${ }^{22}$. Quant au Beaujolais, il traverse une crise grave, conséquence de pratiques laxistes dans la deuxième moitié $\mathrm{du} \mathrm{XX}^{\mathrm{e}}$ siècle. Nombre d'exploitations sont abandonnées. $\mathrm{Ne}$ faudrait-il pas autoriser l'usage de cépages de meilleure qualité que le gamay qui réclame pour donner de bons vins limitation des rendements et méthodes rigoureuses de vinification? La syrah donne des vins merveilleux $80 \mathrm{~km}$ plus au sud, dans la vallée $\mathrm{du}$ Rhône: certains vignerons audacieux du Beaujolais pratiquent des essais actuellement et qui pourrait les en blâmer? C'est peut-être là le moyen de sauver ce vignoble par trop déconsidéré, malgré l'excellence de certains domaines. Les cépages ont voyagé à travers les terroirs de la France, de l'Europe et du monde depuis l'Antiquité. Pourquoi vouloir en interdire aujourd'hui la mobilité ?

Il est sans doute utile de réfléchir à un retour à la diversité de l'encépagement en remettant en culture les plus intéressantes des variétés disparues ou réduites à quelques niches. Ce serait d'autant plus utile que certaines sont sans doute bien adaptés 
à l'évolution climatique actuelle ${ }^{23}$. Il est enfin absurde de s'interdire la recherche sur les modifications génétiques, comme c'est désormais le cas en France et dans toute l'Europe, d'autant que l'on connaît l'extrême variabilité génétique naturelle de beaucoup de cépages. Une solution autorisée n'est encore que faiblement utilisée : l'édition du génome. Elle pourrait permettre d'améliorer la résistance à certains épisodes climatiques, comme la sécheresse, et surtout, aux maladies et, par conséquent, d'éviter les traitements chimiques les plus agressifs ${ }^{24}$.

\section{Conclusion}

Rappelons que la France est le pays d'Europe et même de l'OCDE où l'encadrement de l'État touche le plus grand nombre d'aspects de la vie des citoyens. C'est particulièrement vrai dans le domaine agricole. Le principe de précaution a renforcé cette tendance et la prise de risque est considérée par les dirigeants du pays, comme par les acteurs de base, comme un danger potentiellement mortel. Il est temps de desserrer la réglementation viticole et de promouvoir une confrontation plus franche entre les projets des vignerons et le marché. Personne n'aura rien à y perdre, surtout si l'État (l'INAO) continue à garantir, ce qui est pleinement son rôle, que le contenu des bouteilles commercialisées est conforme à leurs étiquettes. Il y a bien des régions où l'on pourrait autoriser des plantations nouvelles, aux risques et périls des vignerons, comme c'est le cas dans la plupart des pays viticoles du monde, hors de l'Europe. L'origine et la loyauté doivent demeurer des garanties absolues pour les consommateurs. Pour le reste, la liberté ne pourra que donner des ailes aux imaginations vigneronnes et aux amateurs la faculté de peser sur l'art de faire du vin et d'acheter ou non les vins qui leur sont proposés. À défaut de cet assouplissement, gageons que l'INAO, usé jusqu'à la corde, ne vivra pas jusqu'à son centenaire.

\section{BIBLIOGRAPHIE}

Amoreau, Jean-Pierre, Plus pur que l'eau, Paris, Fayard, 2019.

Arnaud, Patrick, Blanc, Michel, Pichery, Marie-Claude (dir.), Les appellations viticoles : leur histoire, leur avenir, Clémencey, Terres en Vues, 2019.

Barret, Philippe et Bouin, Philippe, Appellations, la qualité en danger, Le Rouge \& le Blanc, 120, printemps 2016, pp. 38-44.

Bernaulte, Laura, Liber Pater 2015, le vin le plus cher au monde, terredevins.com, 16 juillet 2019.

Corbeyran et Horne, Liber Pater. Le goût du vin retrouvé, Grenoble, Glénat, 2019.

Cukierman, Jeremy et al., Quel vin pour demain? Le vin face aux défis climatiques, Paris, Dunod, 2021.

Enault, Marianne, Vin : les cépages oubliés défient le climat, Le Journal du Dimanche, 16 mai 2021, p. 25.

Gasparotto, Laure, Jean-Michel Deiss, l'homme qui parle avec sa vigne, Le Monde, $1^{\text {er }}$ juillet 2018. 
Labbé, Thomas, À propos d'une nouvelle découverte : quelques réflexions sur l'apparition du pinot dans les archives bourguignonnes (1366), Crescentis : Revue internationale d'histoire de la vigne et $d u$ vin (en ligne), 2, 2019.

Lautun, Aude, Châteauneuf-du-Pape. Son terroir, sa dégustation, Paris, Flammarion, 2001.

Legouy, Boulanger et al. (dir.), Terre des Hommes, terres du vin, Tours, Presses universitaires François-Rabelais, 2021.

Lucand, Christophe, Comment la France a révolutionnéle monde du vin. La naissance des appellations d'origine, Paris, Dunod, 2019.

Mitjaville, François, La lutte intégrée passe aussi par la génétique de la vigne, blog biotechnologiesvegetales.com, 21 mars 2019.

Perrin, Jacques, Trente années de Mas de Daumas Gassac, 1000 plateaux. Le blog de Jacques Perrin (cavesa.ch), 6 mars 2014.

Pitte, Jean-Robert, Remarques sur la formation du vignoble du Bugey, Acta geographica, 32, 1977, pp. 59-65.

Pitte, Jean-Robert (dir.), Les vins de l'impossible, Grenoble, Glénat, 1990.

Pitte, Jean-Robert, Le désir du vin à la conquête du monde, Paris, Fayard, 2009.

Pitte, Jean-Robert (dir.), Le bon vin entre terroir, savoir-faire et savoir-boire, Actualité de la pensée de Roger Dion, Paris, CNRS-Éditions, 2010.

Pitte, Jean-Robert, Dictionnaire amoureux de la Bourgogne, Paris, Plon 2015.

Pitte, Jean-Robert, Éric de Rothschild et Lafite, une affinité élective, dans Julien Gacon et Aurélie Labruyère (dir.), Esthétique du vin. Conversations pour amateurs, Grenoble, Glénat, 2021, pp. 29-50.

Plageoles, Robert, Vignes premières, vignes de demain, Montbéliard, Tonnerre de l'Est, 2020.

Reynaud, Stéphane, Les guerres picrocholines de Saint-Émilion, Le Figaro, 17-19 juillet 2021, p. 21. Rodarie, Alix, Château d'Yquem s'affranchit de l'appellation Sauternes, idealwine.net, 7 novembre 2013.

Simmat, Benoist, Ces vignerons qui disent bye bye aux appellations, La Revue du Vin de France, 652, juillet-août 2021, pp. 38-44.

Torres, Olivier, La guerre des vins : l'affaire Mondavi. Mondialisation et terroirs, Paris, Dunod, 2005.

Vigneron, Thomas, En Alsace, Jean-Michel Deiss réinvente les vins de terroir et appelle au retour à la complantation, Pioche! (en ligne piochemag.fr), 8 février 2021.

Wolikow, Claudine, Le recours aux usages locaux, loyaux et constants : de l'acquis au mythe (1908-1935...), Territoires du vin (en ligne), 12/2021 et dans François Legouy et al., 2021, pp. 119-129.

\section{NOTES}

1. La question de la promotion du pinot par le duc de Bourgogne Philippe le Hardi en 1395 a été récemment affinée par Thomas Labbé (2019).

2. Aude Lutun, 2001, p. 14.

3. Créateur avec Curnonsky en 1933 de l'Académie du Vin de France dont l'objet est de promouvoir les authentiques vins de terroir et de vignerons. 
4. Cité par Claudine Wolikow, 2021.

5. Cité par Stéphane Reynaud, 2021.

6. Jean-Robert Pitte, 2021, p. 36.

7. Alix Rodarie, 2013.

8. Jean-Robert Pitte, 2015, pp. 664-666.

9. Jean-Pierre Amoreau, 2019.

10. Jean-Robert Pitte (dir.), 1990.

11. Hommage à René Renou, Presse-vin.com, 17, juillet 2006, p. 4.

12. Que je présidais à cette époque.

13. Vitisphère, 12 novembre 2019.

14. Environ 6000 dans le monde dont 13 occupent le tiers des surfaces plantées et 33, la moitié (OIV, 2017). Jeremy Cukierman et al., 2021, p. 49.

15. Jean-Robert Pitte, 1977.

16. Laure Gasparotto, 2018, Thomas Vigneron, 2021.

17. Olivier Torres, 2005, Jacques Perrin, 2014.

18. Philippe Barret et Philippe Bouin, 2016, Benoist Simmat, 2021.

19. Robert Plageoles, 2020.

20. Laura Bernaulte, 2019.

21. Corbeyran et Horne, 2019.

22. Marianne Enault, 2021.

23. C'est l'opinion fermement défendue par l'ampélographe suisse José Vouillamoz, Jeremy Cukierman et al., 2021, pp. 51-56.

24. François Mitjaville, 2019.

\section{RÉSUMÉS}

Élaborée à partir du début du XX $\mathrm{X}^{\mathrm{e}}$ siècle, toute la législation française concernant les $\mathrm{AOC}$, repose sur un triptyque: la fidélité des pratiques viti-vinicoles aux «usages locaux, loyaux et constants ». La recherche d'une typicité liée à un terroir et la rigoureuse provenance des vins d'un territoire délimité indiqué sur l'étiquette demeurent d'actualité. En revanche, la fidélité aux traditions anciennes que recouvre le vocable «constant » mérite d'être revisitée à l'heure de la mondialisation et des évolutions climatiques.

Developed from the beginning of the 20th century, all French legislation concerning AOC is based on a triptych: the fidelity of wine-making practices to «local, loyal and constant uses». The search for a typicity linked to a terroir and the rigorous provenance of the wines of a delimited territory indicated on the label remain current. On the other hand, fidelity to the ancient traditions of the term "constant" deserves to be revisited at a time of globalization and climate change.

\section{INDEX}

Keywords : Designation of origin, constant uses, terroir, classification, typicity

Mots-clés : Appellation d'origine, usages constants, terroir, classement, typicité 
AUTEUR

JEAN-ROBERT PITTE

de l'Institut 